\title{
Diallel Analysis for Combining Ability Studies in Okra [Abelmoschus esculentus (L.) Moench] for Yield and Quality Parameters
}

\author{
A. Shwetha ${ }^{1 *}$, R. Mulge ${ }^{2}$, S. Evoor ${ }^{3}$, V. Kantharaju ${ }^{4}$ and D.A. Masuti ${ }^{5}$ \\ ${ }^{1}$ Department of Vegetable Science, ${ }^{4}$ Department of Plant Pathology, ${ }^{5}$ Department of Seed \\ Science and Technology, KRCCH, Arabhavi-591218, Karnataka, India \\ ${ }^{2}$ College of Horticulture, Bidar - 585 401, Karnataka, India \\ ${ }^{3}$ Department of Vegetable Science, COH, Bagalkot-587104, Karnataka, India
}

*Corresponding author

\section{A B S T R A C T}

\begin{tabular}{|l|}
\hline Ke y w o r d s \\
$\begin{array}{l}\text { General combining } \\
\text { ability, Specific } \\
\text { combining ability, } \\
\text { Half diallel }\end{array}$ \\
\hline Article Info \\
\hline $\begin{array}{l}\text { Accepted: } \\
\text { 16 August } 2018 \\
\text { Available Online: } \\
\text { 10 September } 2018\end{array}$ \\
\hline
\end{tabular}

Key w o r d s

General combining ability, Specific combining ability, Half diallel

Accepted:

Available Online:

\begin{abstract}
Eight parental lines of okra and their 28 F1 hybrids obtained from half diallel were studied to identify the good general and specific combiners for yield and quality parameters. Both general a combining ability (GCA) and specific combining ability (SCA) variances were highly significant for all the characters indicating the importance of both additive and nonadditive gene actions. The parents KO1608 and KO1606 were identified as good general combiners for number of fruits per plant, yield per plant, yield per plot and yield per hectare. The parent KO1608 was good general combiner for fruit weight and number of ridges on fruit surface and the parent KO1606 was identified as good general combiner for average fruit weight. The crosses KO1601 x KO1605 and KO1603 x KO1606 were identified as good specific combiners for average fruit weight, yield per plant, yield per plot and yield per hectare. The maximum sca effects were observed in the crosses KO1601 x KO1604 and KO1606 x KO1607 for number of fruits per plant and fruit length respectively. The cross KO1602 x KO1605 exhibited highest sca effects for fruit diameter and number of seeds per fruit.
\end{abstract}

\section{Introduction}

Okra [Abelmoschus esculentus (L.) Moench] is a fast growing annual which has captured a prominent position among the vegetables and is commonly known as bhendi or lady's finger in India. India is the largest producer of okra in the world with an annual production of 63.46 lakh tonnes from an area of 5.32 lakh hectare with a productivity of 11.9 tonnes per hectare (Anon., 2014). Okra is specially valued for its tender, delicious green fruits which are cooked, canned and consumed in various forms in different parts of the country. Combining ability analysis helps in the evaluation of inbreds in terms of their genetic value and the selection of suitable parents for hybridization and helps in the identification of superior cross combination, which ultimately helps in deciding about exploitation of 
heterosis using the specific cross combination. Clear- cut knowledge of the type of gene action and magnitude and composition of genetic variance is of fundamental importance to a plant breeder. Diallel analysis technique developed by Jinks and Hayman (1953) has been extensively used to estimate GCA and SCA variances and to understand the nature of gene action involved in the expression of various quantitative traits. Hence, the present investigation was undertaken to obtain the information on combining ability of eight genotypes of okra (Abelmoschus esculentus L.) for yield and quality parameters.

\section{Materials and Methods}

The investigation on combining ability studies in okra was carried out at the Department of Vegetable Science, K.R.C. College of Horticulture, Arabhavi, Gokak Taluk, Belagavi district of Karnataka state. The experimental material comprised of 8 parents (KO1601, KO1602, KO1603, KO1604, KO1605, KO1606, KO1607 and KO1608), which were collected from the department itself and their $28 \mathrm{~F} 1$ hybrids. Each of the 8 parents crossed among each other in half diallel fashion without reciprocal crosses to derive $28 \mathrm{~F}_{1}$ hybrids. The experiment was laid out in randomized block design with two replications. Each treatment or a genotype in each replication was represented by one row each accommodating 20 plants at a row to row spacing of $60 \mathrm{~cm}$ and $30 \mathrm{~cm}$ from plant to plant. Five plants were randomly selected for each genotype from each replication and evaluated for the quantitative characters and the replicated mean values of various characters of parents and hybrids were subjected to half diallel analysis.

\section{Results and Discussion}

The variance due to genotypes (crosses and parents) was highly significant (at $\mathrm{p}=0.01$ ) for all the yield and quality parameters, viz., fruit length, fruit diameter, average fruit weight, number of fruits per plant, total yield per plant, yield per plot, yield per hectare, number of ridges on fruit surface and number of seeds per fruit. Parents differed significantly among themselves for all the yield and quality parameters studied except for fruit diameter and average fruit weight. There was highly significant (at $\mathrm{p}=0.01$ ) difference among the crosses for all the yield and quality parameters studied. Variance due to parents vs crosses was significant for fruit length, average fruit weight, number of fruits per plant and number of ridges on fruit surface and for all other parameters variance due to parents vs crosses was not significant (Table 1).

The mean sum of squares due to SCA and GCA were found highly significant for all the parameters. Ratio of general combining ability variance (GCA) to specific combining ability variance (SCA) is an indication of predominance of additive or non-additive genetic variance. GCA to SCA ratio (Table 2) was low for the traits fruit diameter (Paul et al., 2017 and Bhatt et al., 2015), number of ridges on fruit surface (Akotkar et al., 2014), fruit length (Paul et al., 2017), number of seeds per fruit (Laxman et al., 2013) and average fruit weight (Bhatt et al., 2015, Nagesh et al., 2014 and Laxman et al., 2013) indicating preponderance of non-additive gene action and hence these traits can be improved through recurrent selection schemes or heterosis breeding. Non-additive component of genetic variance was slightly higher than additive components for yield per hectare (Reddy et al., 2013), total yield per plant (Reddy et al., 2013) and number of fruits per plant (Jonah et al., 2015) Hence, direct selection or recurrent selection schemes can be employed for improvement of these traits. General combining ability effects and specific combining ability effects for various traits are presented in Tables 3 to 5 . 
Table.1 Analysis of variance (mean sum of squares) of diallel analysis for various characters in okra

\begin{tabular}{|c|c|c|c|c|c|c|c|}
\hline \multirow{2}{*}{$\begin{array}{r}\text { Sl. } \\
\text { No. } \\
\end{array}$} & Character & Replications & Genotypes & Parents & Crosses & Parents vs Crosses & Error \\
\hline & Degrees of freedom & 1 & 35 & 7 & 27 & 1 & 35 \\
\hline a. & \multicolumn{7}{|l|}{ Yield parameters } \\
\hline 1. & Fruit length $(\mathrm{cm})$ & 0.80 & $2.94 * *$ & $3.23 * *$ & $2.83 * *$ & $3.76^{*}$ & 0.89 \\
\hline 2. & Fruit diameter (mm) & 0.75 & $2.17 * *$ & $1.53 \mathrm{NS}$ & $2.36^{* *}$ & $1.69 \mathrm{NS}$ & 0.75 \\
\hline 3. & Average fruit weight $(\mathrm{g})$ & 1.85 & $5.31 * *$ & $1.31 \mathrm{NS}$ & $6.36 * *$ & $4.98^{*}$ & 1.03 \\
\hline 4. & Number of fruits per plant & 5.58 & $4.44 * *$ & $6.77 * *$ & $3.65 * *$ & $9.43 * *$ & 0.64 \\
\hline 5. & Total yield per plant (kg) & 0.001 & $0.003 * *$ & $0.002 * *$ & $0.003 * *$ & $0.00 \mathrm{NS}$ & 0.001 \\
\hline 6. & Yield per plot $(\mathrm{kg})$ & 0.40 & $0.87 * *$ & $0.73 * *$ & $0.94 * *$ & $0.07 \mathrm{NS}$ & 0.16 \\
\hline 7. & Yield per hectare (t) & 3.14 & $6.73 * *$ & $5.62 * *$ & $7.25 * *$ & $0.55 \mathrm{NS}$ & 1.26 \\
\hline b. & \multicolumn{7}{|l|}{ Quality parameters } \\
\hline 8. & Number of ridges on fruit surface & 0.03 & $0.08 * *$ & $0.17 * *$ & $0.06 * *$ & $0.05 *$ & 0.01 \\
\hline 9. & Number of seeds per fruit & 7.74 & $82.12 * *$ & $53.63 * *$ & $92.46 * *$ & $2.03 \mathrm{NS}$ & 8.42 \\
\hline
\end{tabular}

$*$ and $* *$ indicate significance of values at $\mathrm{p}=0.05$ and $\mathrm{p}=0.01$, respectively. NS: Non significant, DAS: Days after sowing.

Table.2 Variance due to general and specific combining ability for yield and quality parameters in okra

\begin{tabular}{|c|c|c|c|c|c|c|c|}
\hline \multirow{2}{*}{$\begin{array}{c}\text { Sl. } \\
\text { No. }\end{array}$} & \multirow[t]{2}{*}{ Character } & \multicolumn{3}{|c|}{ Mean sum of square } & \multirow[t]{2}{*}{$\sigma_{g}^{2}$} & \multirow[t]{2}{*}{$\sigma_{s}^{2}$} & \multirow[t]{2}{*}{$\sigma_{\mathrm{g}}^{2}: \sigma_{\mathrm{s}}^{2}$} \\
\hline & & GCA & SCA & Error & & & \\
\hline 1. & Fruit length $(\mathrm{cm})$ & $2.053 * *$ & $1.324 * *$ & 0.445 & 0.161 & 0.879 & 0.183 \\
\hline 2. & Fruit diameter (mm) & $2.327 * *$ & $0.779 *$ & 0.377 & 0.195 & 0.402 & 0.485 \\
\hline 3. & Average fruit weight (g) & $2.794 * *$ & $2.621 * *$ & 0.516 & 0.228 & 2.104 & 0.108 \\
\hline 4. & Number of fruits per plant & $6.335 * *$ & $1.193 * *$ & 0.319 & 0.602 & 0.874 & 0.688 \\
\hline 5. & Total yield per plant (kg) & $0.004 * *$ & $0.001 * *$ & 0.0002 & 0.0004 & 0.0005 & 0.795 \\
\hline 6. & Yield per plot $(\mathrm{kg})$ & $1.265^{* *}$ & $0.229 * *$ & 0.082 & 0.118 & 0.147 & 0.804 \\
\hline 7. & Yield per hectare $(\mathrm{t})$ & $9.771 * *$ & $1.766^{* *}$ & 0.630 & 0.914 & 1.136 & 0.805 \\
\hline 8. & Number of ridges on fruit surface & $0.087 * *$ & $0.033 * *$ & 0.005 & 0.008 & 0.027 & 0.299 \\
\hline 9. & Number of seeds per fruit & $56.444 * *$ & $37.211 * *$ & 4.212 & 5.223 & 32.998 & 0.158 \\
\hline
\end{tabular}

$*$ and $* *$ indicate significance of values at $\mathrm{p}=0.05$ and $\mathrm{p}=0.01$, respectively. 
Table.3 General combining ability effects of parents for fruit characters, yield parameters, number of ridges on fruit surface and number of seeds per fruit in okra

\begin{tabular}{|c|c|c|c|c|c|c|c|c|c|c|}
\hline $\begin{array}{l}\text { Sl. } \\
\text { No. }\end{array}$ & Parents & $\begin{array}{l}\text { Fruit } \\
\text { length }\end{array}$ & $\begin{array}{c}\text { Fruit } \\
\text { diameter }\end{array}$ & $\begin{array}{l}\text { A verage } \\
\text { fruit } \\
\text { weight }\end{array}$ & $\begin{array}{l}\text { Number } \\
\text { of fruits } \\
\text { per plant }\end{array}$ & $\begin{array}{l}\text { Yield per } \\
\text { plant }\end{array}$ & $\begin{array}{c}\text { Yield per } \\
\text { plot }\end{array}$ & $\begin{array}{l}\text { Yield per } \\
\text { hectare }\end{array}$ & $\begin{array}{l}\text { Number of } \\
\text { ridges on } \\
\text { fruit surface }\end{array}$ & $\begin{array}{l}\text { Number of } \\
\text { seeds per } \\
\text { fruit }\end{array}$ \\
\hline 1. & KO1601 & -0.179 & 0.109 & $-0.816 * *$ & $-0.327 *$ & $-0.017 * *$ & $-0.280 * *$ & $-0.779 * *$ & $0.187 * *$ & 0.520 \\
\hline 2. & KO1602 & -0.195 & $-0.954 * *$ & $-0.658 * *$ & $0.780 * *$ & 0.007 & 0.116 & 0.320 & $0.047 *$ & $-2.220 * *$ \\
\hline 3. & KO1603 & $0.851 * *$ & 0.174 & $0.620 * *$ & $-0.838 * *$ & $-0.011 *$ & $-0.192 *$ & $-0.533^{*}$ & -0.013 & $4.300 * *$ \\
\hline 4. & KO1604 & $-0.467 *$ & 0.234 & 0.073 & $-1.002 * *$ & $-0.027 * *$ & $-0.456 * *$ & $-1.269 * *$ & 0.027 & $-3.280 * *$ \\
\hline 5. & KO1605 & -0.099 & 0.144 & 0.029 & -0.281 & -0.008 & -0.144 & -0.399 & $-0.052 *$ & $1.870 * *$ \\
\hline 6. & KO1606 & $-0.487 *$ & -0.140 & $0.680 * *$ & $0.625 * *$ & $0.031 * *$ & $0.521 * *$ & $1.447 * *$ & -0.002 & 0.450 \\
\hline 7. & KO1607 & 0.208 & -0.277 & 0.071 & -0.187 & -0.004 & -0.067 & -0.184 & $-0.073 * *$ & -0.920 \\
\hline 8. & KO1608 & 0.368 & $0.711 * *$ & -0.001 & $1.228 * *$ & $0.030 * *$ & $0.502 * *$ & $1.396 * *$ & $-0.123 * *$ & -0.720 \\
\hline & SEm \pm & 0.197 & 0.182 & 0.213 & 0.167 & 0.005 & 0.085 & 0.235 & 0.021 & 0.607 \\
\hline & D at $5 \%$ & 0.466 & 0.429 & 0.503 & 0.339 & 0.011 & 0.200 & 0.555 & 0.049 & 1.435 \\
\hline & $D$ at $1 \%$ & 0.690 & 0.636 & 0.744 & 0.455 & 0.017 & 0.296 & 0.822 & 0.073 & 2.124 \\
\hline
\end{tabular}

$*$ and $* *$ indicates significance of value at $\mathrm{p}=0.05$ and $\mathrm{p}=0.01$, respectively 
Table.4 Specific combining ability effects of crosses for fruit parameters in okra

\begin{tabular}{|c|c|c|c|c|c|}
\hline $\begin{array}{l}\text { Sl. } \\
\text { No. }\end{array}$ & Crosses & Fruit length & $\begin{array}{c}\text { Fruit } \\
\text { diameter }\end{array}$ & $\begin{array}{c}\text { Average } \\
\text { fruit weight }\end{array}$ & $\begin{array}{l}\text { Number of } \\
\text { fruits per plant }\end{array}$ \\
\hline 1 & KO1601 x KO1602 & -0.061 & -0.140 & $-2.386 * *$ & $1.378 * *$ \\
\hline 2 & KO1601 x KO1603 & -0.136 & 0.498 & -0.074 & -0.554 \\
\hline 3 & KO1601 x KO1604 & -0.204 & -0.308 & $-1.472 *$ & $2.460 * *$ \\
\hline 4 & KO1601 x KO1605 & $-2.062 * *$ & -0.732 & $3.012 * *$ & 0.939 \\
\hline 5 & KO1601 x KO1606 & 0.941 & 0.657 & $-2.604 * *$ & -0.867 \\
\hline 6 & KO1601 x KO1607 & -1.044 & $1.298^{*}$ & 0.980 & -0.355 \\
\hline 7 & KO1601 x KO1608 & 0.256 & -0.239 & 0.162 & $1.230 *$ \\
\hline 8 & KO1602 x KO1603 & 0.335 & $-1.854 * *$ & -0.997 & $-1.461 * *$ \\
\hline 9 & KO1602 x KO1604 & $-1.244^{*}$ & -0.255 & 0.730 & -0.397 \\
\hline 10 & KO1602 x KO1605 & 1.214 & $1.446^{*}$ & $1.439 *$ & 0.332 \\
\hline 11 & KO1602 x KO1606 & $-1.688 * *$ & -0.145 & -0.002 & -0.324 \\
\hline 12 & KO1602 x KO1607 & 1.162 & $1.231^{*}$ & $-2.448 * *$ & 0.888 \\
\hline 13 & KO1602 x KO1608 & -0.378 & $-1.381 *$ & 0.534 & -0.737 \\
\hline 14 & KO1603 x KO1604 & -0.689 & 0.548 & -0.178 & 0.271 \\
\hline 15 & KO1603 x KO1605 & 0.769 & 0.098 & 0.086 & 0.350 \\
\hline$\overline{16}$ & KO1603 x KO1606 & 0.702 & 1.117 & $1.865 * *$ & $1.930 * *$ \\
\hline 17 & KO1603 x KO1607 & -0.164 & -0.396 & 1.195 & -0.244 \\
\hline 18 & KO1603 x KO1608 & -0.004 & 0.321 & 0.261 & 0.891 \\
\hline 19 & KO1604 x KO1605 & $1.695 * *$ & 0.003 & $-2.822 * *$ & -0.536 \\
\hline 20 & KO1604 x KO1606 & -0.341 & $-1.158 *$ & $2.032 * *$ & -0.492 \\
\hline 21 & KO1604 x KO1607 & $1.423 *$ & -0.067 & -1.179 & -0.330 \\
\hline 22 & KO1604 x KO1608 & 0.453 & 0.641 & -0.377 & 0.155 \\
\hline 23 & KO1605 x KO1606 & 0.376 & -0.143 & $-3.074 * *$ & 0.867 \\
\hline 24 & KO1605 x KO1607 & -1.149 & $-1.246^{*}$ & 1.250 & $-1.151^{*}$ \\
\hline 25 & KO1605 x KO1608 & -0.820 & 0.756 & -0.463 & 0.414 \\
\hline 26 & KO1606 x KO1607 & $2.209 * *$ & 0.328 & 0.844 & 0.743 \\
\hline 27 & KO1606 x KO1608 & 1.054 & 1.100 & 0.241 & $-1.222 *$ \\
\hline 28 & KO1607 x KO1608 & 0.813 & 0.312 & -0.490 & $1.240 *$ \\
\hline & SEm \pm & 0.605 & 0.557 & 0.652 & 0.512 \\
\hline & CD at $5 \%$ & 1.241 & 1.143 & 1.337 & 1.040 \\
\hline & CD at $1 \%$ & 1.675 & 1.543 & 1.805 & 1.395 \\
\hline
\end{tabular}

*and ${ }^{* *}$ indicate significance of values at $\mathrm{p}=0.05$ and $\mathrm{p}=0.01$, respectively. 
Table.5 Specific combining ability effects of crosses for yield and quality parameters in okra

\begin{tabular}{|c|c|c|c|c|c|c|}
\hline $\begin{array}{l}\text { Sl. } \\
\text { No. }\end{array}$ & Crosses & $\begin{array}{l}\text { Yield per } \\
\text { plant }\end{array}$ & $\begin{array}{l}\text { Yield per } \\
\text { plot }\end{array}$ & $\begin{array}{l}\text { Yield per } \\
\text { hectare }\end{array}$ & $\begin{array}{l}\text { Number of } \\
\text { ridges on } \\
\text { fruit surface }\end{array}$ & $\begin{array}{l}\text { Number } \\
\text { of seeds } \\
\text { per fruit }\end{array}$ \\
\hline 1 & KO1601 x KO1602 & -0.028 & -0.462 & -1.290 & $-0.460 * *$ & $-7.611 * *$ \\
\hline 2 & KO1601 x KO1603 & -0.016 & -0.275 & -0.761 & -0.100 & $-5.131 *$ \\
\hline 3 & KO1601 x KO1604 & -0.024 & -0.400 & -1.115 & -0.040 & $5.749 * *$ \\
\hline 4 & KO1601 x KO1605 & $0.066 * *$ & $1.112 * *$ & $3.089 * *$ & -0.060 & -1.601 \\
\hline 5 & KO1601 x KO1606 & $-0.036 *$ & $-0.613 *$ & $-1.702 *$ & -0.010 & $14.219 * *$ \\
\hline 6 & KO1601 x KO1607 & 0.002 & 0.020 & 0.055 & $-0.240 * *$ & $-6.811 * *$ \\
\hline 7 & KO1601 x KO1608 & 0.011 & 0.181 & 0.505 & $0.310 * *$ & -2.111 \\
\hline 8 & KO1602 x KO1603 & $-0.047 * *$ & $-0.801 * *$ & $-2.225 * *$ & -0.060 & $6.509 * *$ \\
\hline 9 & KO1602 x KO1604 & 0.005 & 0.084 & 0.231 & 0.000 & -1.611 \\
\hline 10 & KO1602 x KO1605 & 0.028 & 0.472 & 1.310 & 0.080 & $-4.461 *$ \\
\hline 11 & KO1602 x KO1606 & -0.011 & -0.188 & -0.516 & 0.130 & -0.341 \\
\hline 12 & KO1602 x KO1607 & $-0.038 *$ & $-0.641 *$ & $-1.779 *$ & $0.300 * *$ & $9.129 * *$ \\
\hline 13 & KO1602 x KO1608 & 0.023 & 0.391 & 1.096 & $-0.150^{*}$ & -0.471 \\
\hline 14 & KO1603 x KO1604 & 0.009 & 0.161 & 0.449 & -0.040 & 0.269 \\
\hline 15 & KO1603 x KO1605 & 0.011 & 0.194 & 0.534 & $0.140 *$ & $4.519 *$ \\
\hline 16 & KO1603 x KO1606 & $0.037 *$ & $0.629 *$ & $1.743 *$ & -0.110 & $-13.961 * *$ \\
\hline 17 & KO1603 x KO1607 & 0.013 & 0.226 & 0.634 & $-0.140 *$ & $9.709 * *$ \\
\hline 18 & KO1603 x KO1608 & 0.019 & 0.323 & 0.889 & -0.090 & -1.091 \\
\hline 19 & KO1604 x KO1605 & $-0.046 * *$ & $-0.776 * *$ & $-2.150 * *$ & $-0.200 * *$ & -0.101 \\
\hline 20 & KO1604 x KO1606 & 0.018 & 0.304 & 0.849 & $0.150 *$ & -2.581 \\
\hline 21 & KO1604 x KO1607 & -0.018 & -0.304 & -0.845 & $0.220 * *$ & -0.211 \\
\hline 22 & KO1604 x KO1608 & -0.006 & -0.102 & -0.280 & 0.070 & -1.611 \\
\hline 23 & KO1605 x KO1606 & -0.027 & -0.464 & -1.287 & 0.030 & -1.331 \\
\hline 24 & KO1605 x KO1607 & -0.005 & -0.071 & -0.200 & -0.100 & $-8.661 * *$ \\
\hline 25 & KO1605 x KO1608 & -0.011 & -0.180 & -0.495 & -0.050 & $6.339 * *$ \\
\hline 26 & KO1606 x KO1607 & 0.022 & 0.374 & 1.034 & $0.150 *$ & $4.259 *$ \\
\hline 27 & KO1606 x KO1608 & 0.014 & 0.235 & 0.654 & -0.100 & 2.959 \\
\hline 28 & KO1607 x KO1608 & 0.005 & 0.093 & 0.260 & -0.030 & $-6.471 * *$ \\
\hline & SEm \pm & 0.015 & 0.259 & 0.259 & 0.064 & 1.861 \\
\hline & CD at $5 \%$ & 0.031 & 0.532 & 0.532 & 0.132 & 3.818 \\
\hline & CD at $1 \%$ & 0.042 & 0.718 & 0.718 & 0.178 & 5.156 \\
\hline
\end{tabular}

*and $* *$ indicate significance of values at $\mathrm{p}=0.05$ and $\mathrm{p}=0.01$, respectively.

For fruit length, only one parent KO1603 (0.851) exhibited significantly positive gca effects and three crosses showed significant positive sca effects. The maximum sca effects was observed in the cross KO1606 x KO1607 (2.209) followed by KO1604 x KO1605
(1.695) and KO1604 x KO1607 (1.423). For fruit diameter, only one parent KO1608 (0.711) exhibited significantly positive gca effects and three crosses showed significant positive sca effects. The maximum sca effects was observed in the cross KO1602 x KO1605 
(1.446) followed by KO1601 x KO1607 (1.298) and KO1602 x KO1607 (1.231).

For average fruit weight, two parents viz., KO1606 (0.68) and KO1603 (0.62) exhibited significant positive gca effects and four crosses showed significantly positive sca effects. The maximum sca effects was observed in the cross KO1601 x KO1605 (3.012) followed by KO1604 x KO1606 (2.032), KO1603 x KO1606 (1.865) and KO1602 x KO1605 (1.439).

For number of fruits per plant, three parents exhibited significantly positive gca effects and the maximum gca effects was observed in the parent KO1608 (1.228) followed by KO1602 (0.78) and KO1606 (0.625) and five crosses exhibited significant positive sca effects. The maximum sca effects was observed in the cross KO1601 x KO1604 (2.46) followed by KO1603 x KO1606 (1.93).

For total yield per plant, two parents viz., KO1606 (0.031) and KO1608 (0.030) exhibited significantly positive gca effects and only two crosses showed significant positive sca effects. The maximum sca effects was observed in the cross KO1601 x KO1605 (0.066) followed by KO1603 x KO1606 (0.037).

For total yield per plot, two parents viz., KO1606 (0.521) and KO1608 (0.502) exhibited significantly positive gca effects and only two crosses exhibited significant positive sca effects. The maximum sca effects was observed in the cross KO1601 x KO1605 (1.112) followed by KO1603 x KO1606 $(0.629)$.

For total yield per hectare, only two parents exhibited significantly positive gca effects and the maximum gca effects was observed in the parent KO1606 (1.447) followed by KO1608 (1.396) and two crosses exhibited significant positive sca effects. The maximum sca effects was observed in the cross KO1601 x KO1605 (3.089) followed by KO1603 x KO1606 (1.743).

The parents and crosses with negative combining ability effects (gca and sca) are preferred. for number of ridges on fruit surface, three parents exhibited significantly negative gca effects and the maximum negative gca effects was observed in the parent KO1608 (-0.123) followed by KO1607 (-0.073) and KO1605 (-0.052). Among 28 crosses, five crosses exhibited significant negative sca effects. The maximum negative sca effects was observed in the cross KO1601 x KO1602 (-0.46) followed by KO1601 x KO1607 (-0.24).

For number of seeds per fruit, two parents showed significantly positive gca effects and the maximum gca effects was observed in the parent KO1603 (4.30) followed by KO1605 (1.87) and eight crosses exhibited significant positive sca effects. The maximum sca effects was observed in the cross KO1601 x KO1606 (14.219) followed by KO1603 x KO1607 (9.709).

Comprehensive assessment of parents by considering gca effects of nine characters studied has resulted into identification of parents viz., KO16058 and KO1606 as good combiners and parents viz., KO1601 and KO1604 were identified as poor combiners for most of the characters. The crosses KO1601 x KO1605 and KO1603 x KO1606 were identified as good specific combiners for average fruit weight, yield per plant, yield per plot and yield per hectare.

The parents KO1608 and KO1606 are the good general combiners for number of fruits per plant and yield per hectare and these can be used in identifying superior new heterotic combinations. Fruit length, fruit diameter, 
average fruit weight, number of ridges on fruit surface and number of seeds per fruit are predominantly controlled by non-additive gene action and hence heterosis breeding and recurrent selection can be employed for improvement. Non additive component of genetic variance was slightly higher than additive component for numbers of fruits per plant, total yield per plant and yield per hectare and these characters can be improved through direct selection or recurrent selection schemes.

\section{Acknowledgement}

The author thankful to Dr. Ravindra Mulge, Dean, College of Horticulture, Bidar and Dr. Shashikanth Evoor, Asst. Professor, Department of Vegetable Science, College of Horticulture, Bagalkot for endless support during the course of investigation.

\section{References}

Akotkar, P. K., De, D. K. and Dubey, U. K., 2014, Genetic studies on fruit yield and yield attributes of okra (Abelmoschus esculentus (L.) Moench). Electron. J. Breed., 5(1): 38-44.

Anonymous, 2014, Indian Horticultural Database, 2014. http://www.nhb.gov.in.

Bhatt, J. P., Kathira, K. B., Christian, S. S. and Acharya, R. R., 2015, Combining ability studies in okra (Abelmoschus esculentus (L.) Moench) for yield and its component characters. Electronic $J$. Plant Breed., 6(2): 479-485.

Jinks, J. L. and Hayman, B. I., 1953, The analysis of diallel crosses. Maize Genetics Newsletter, 27: 48 - 54.

Jonah, P. M., Bello, L. L., Kalu, B. A., Omoigui, L. O. and Adeniji, O. T., 2015, Combining ability of yield characters and selection for fruit gelatinization Kumar, S. and Reddy, M. T., 2016a, Combining ability of inbred lines and half-diallel crosses for economic traits in okra (Abelmoschus esculentus (L.) Moench). Jordan J. Agric. Sci., 12(2): 479-497.

Laxman, M., Shanthakumar, G., Thimmanna, P. O., Udaykumar, K., Prakash, G. and Sateesh, A., 2013, Nutritional enhancement for iron content and combining ability studies in newly derived inbred lines of okra (Abelmoschus esculentus Moench L.). Molecular Plant Breed., 4(3) 24-30.

Nagesh, G. C., Mulge, R., Rathod, V., Basavraj, B. L. and Mahaveer, S. M., 2014, Heterosis and combining ability studies in okra (Abelmoschus esculentus (L.) Moench) for yield and yield quality parameters. The Bioscan, 9(4): 17171723.

Paul, T., Desai, R. T. and Choudhary, R., 2017, Genetic architecture, combining ability and gene action study in okra [Abelmoschus esculentus (L.) Moench]. Int. J. Curr. Microbiol. App. Sci., 6(4): 851-858.

\section{How to cite this article:}

Shwetha, A., R. Mulge, S. Evoor, V. Kantharaju and Masuti, D.A. 2018. Diallel Analysis for Combining Ability Studies in Okra [Abelmoschus esculentus (L.) Moench] for Yield and Quality Parameters. Int.J.Curr.Microbiol.App.Sci. 7(09): 2114-2121. doi: https://doi.org/10.20546/ijcmas.2018.709.258 